\title{
DIFFERENTIAL GEOMETRICAL RELATIONS FOR A CLASS OF FORMAL SERIES
}

\author{
ALEXANDR BARANOVITCH \\ National Technical University of Ukraine \\ 37 Peremogy prosp., Kiev, Ukraine
}

\begin{abstract}
An extension of the category of local manifolds is considered. Instead of smooth mappings of neighbourhoods of linear spaces as morphisms we deal with formal operator power series (or formal maps). Analogues of the objects appearing on smooth manifolds and vector bundles (vector fields, sections of a bundle, exterior forms, the de Rham complex, connection, etc.) are considered in this way. All the examinations are carried out in algebraic language, for we do not care about the convergence of formal maps. It may be useful for the investigation of some nonlinear differential equations.
\end{abstract}

$\mathbf{1}^{\circ}$. Principal objects of differential geometry are smooth manifolds, bundles, sections of bundles, and connections. Their local definitions, properties, and relations are formulated in terms of the category whose objects are linear spaces (more exactly, neighbourhoods in linear spaces) and whose morphisms are smooth maps of these neighbourhoods. One can try to extend the category by replacing the morphisms with others. It leads to some reformulation of the theory.

We do such a reformulation in the case when formal functional and operator power series play the role of morphisms ([1], [4]). All the operations are performed not over maps, but over the sets of their Tailor coefficients. The operations prove to be correct because substituting a series without zero term for the arguments of another series one gets that the coefficients of the resulting series are some finite sums. But now one need not make any assumptions about the convergence of the series. Therefore the reformulation is substantial: we turn in fact from analysis to algebra. Particularly, it turns out to be useful for some procedures of calculation of solutions of nonlinear differential equations ([3], [5]).

We call the morphisms described by the sets of coefficients the formal mappings. An associative operation $\circ$ of composition of formal mappings corresponding to the substi-

1991 Mathematics Subject Classification: Primary 58B30; Secondary 53B99.

Supported in part by the International Science Foundation, Grant No U44200, and by the Fundamental Research Foundation of the SCST of the Ukraine

The paper is in final form and no version of it will be published elsewhere. 
tution a series for the arguments of another one, and an operation \# corresponding to the differentiation along a direction are defined.

The latter operation is not associative. However the commutator built on it defines a bracket subjected to the Jacobi identity. The bracket is analogous to the Gerstenhaber bracket $([6])$. With the help of the above mentioned operations we define local manifolds, bundles over them, algebras of differential forms, connections.

Notice that constructions connected with the exponential map of the Lie algebra under consideration: a composite exponential and its inversion - a composite logarithm - are described in [1] in this way.

Another aspect of the work is its connections with formal differential geometry which is considered from the point of view of the modern analysis, where instead of a geometrical object one considers a set of functions on it. There (see [5]) instead of a smooth manifold its formal generalization is considered: a module (of formal mappings) over a Lie algebra (of formal vector fields).

The results described in this publication were announced in [2].

$\mathbf{2}^{\circ}$. Now we introduce the main objects and operations on them.

Let $X, Y$ be linear topological spaces (l.t.s.'s) over a field $\mathbb{K}$ (when it is not important that spaces are topological, we will write simply l.s.). Let $L_{k}(X, Y)$ be 1.t.s. of $k$-linear mappings from $X$ to $Y$, and let $L_{k, s}(X, Y)$ be its subspace consisting of symmetrical mappings, $L_{0, s}(X, Y)=Y$.

Consider the l.s.'s

$$
\mathcal{L}(X, Y)=\prod_{k=1}^{\infty} L_{k}(X, Y), \quad \mathcal{L}_{s}(X, Y)=\prod_{k=0}^{\infty} L_{k, s}(X, Y),
$$

whose elements are infinite sets of the form

$$
a=\left(a_{1}, a_{2}, \ldots, a_{k}, \ldots\right) \in \mathcal{L}(X, Y), \quad f=\left(f_{0}, f_{1}, \ldots, f_{k}, \ldots\right) \in \mathcal{L}_{s}(X, Y) .
$$

They are called formal mappings (f.m.'s).

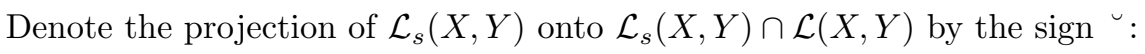

$$
f=\left(f_{0}, f_{1}, \ldots, f_{k}, \ldots\right) \in \mathcal{L}_{s}(X, Y) \Rightarrow \breve{f}=\left(f_{1}, \ldots, f_{k}, \ldots\right) .
$$

Introduce a composition

$$
\begin{gathered}
\circ: \mathcal{L}(Y, Z) \times \mathcal{L}(X, Y) \rightarrow \mathcal{L}(X, Z), \\
(a \circ b)_{k}=\sum_{j=1}^{k} \sum_{k_{1}+\ldots+k_{j}=k} a_{j} \circ\left(\bigotimes_{m=1}^{j} b_{k_{m}}\right) .
\end{gathered}
$$

Proposition 1. The operation o is associative.

Denote an identity mapping of a space $X$ by $I_{X} \in L_{1}(X, X)=L_{1}(X)$. Obviously, the f.m. $i d_{X}=\left(I_{X}, 0, \ldots, 0, \ldots\right) \in \mathcal{L}(X)=\mathcal{L}(X, X)$ is an identity element with respect to the composition. Thus we obtain the category of formal mappings $\mathcal{X}$, whose objects are l.s.'s and $\operatorname{Hom}(X, Y)=\mathcal{L}(X, Y)$. 
Obviously, it is necessary for $a \in \mathcal{L}(X, Y)$ to be invertible that $a_{1}$ is invertible. It is proved to be sufficient too. So, the elements of the set

$$
G(X, Y)=\left\{a \in \mathcal{L}(X, Y) \mid \exists a_{1}^{-1} \in L_{1}(Y, X)\right\}
$$

(and only they) are invertible with respect to the composition. Notice the set $\mathcal{G}(X)=$ $G(X, X)$ is a group with respect to the composition.

An element $\varphi \in \mathcal{L}(X, Y)$ generates a morphism of objects on $X$ into objects on $Y$ being a homomorphism of $\mathcal{L}_{s}(Y, Z)$ into $\mathcal{L}_{s}(X, Z)$ :

$$
\begin{gathered}
\stackrel{s}{\circ}: \mathcal{L}_{s}(Y, Z) \times \mathcal{L}(X, Y) \rightarrow \mathcal{L}_{s}(X, Z), \\
f \stackrel{s}{\circ} \varphi=f_{0} \times \mathrm{S}(\breve{f} \circ \varphi),
\end{gathered}
$$

where $\mathrm{S}$ is the operation of symmetrization of the set of polylinear mappings.

Proposition 2. If $\varphi \in \mathcal{L}(Y, Z), \psi \in \mathcal{L}(X, Y), f \in \mathcal{L}_{s}\left(Z, Z^{\prime}\right)$, then

$$
f \stackrel{s}{\circ}(\varphi \circ \psi)=(f \stackrel{s}{\circ} \varphi) \stackrel{s}{\circ} \psi=\mathrm{S}\left(f_{0} \times(\breve{f} \circ \varphi \circ \psi)\right),
$$

so we can say the symmetrical composition is associative too.

Let us consider, for each element of the group $\mathcal{G}(X)$, the morphism generated by the inverted element. Such a morphism is called a change of variables (or a coordinate transformation). All the changes of variables in $\mathcal{L}_{s}(X, Y)$ form a representation of the group $\mathcal{G}(X)$ in the l.s. $\mathcal{L}_{s}(X, Y)$.

Suppose that, for elements of spaces $Y, Z, V$, a bilinear operation of multiplication . $: Y \times Z \rightarrow V$ is defined. We extend it to the elements of the l.s.'s $\mathcal{L}_{s}(X, Y), \mathcal{L}_{s}(X, Z)$ by

$$
\cdot: \mathcal{L}_{s}(X, Y) \times \mathcal{L}_{s}(X, Z) \rightarrow \mathcal{L}_{s}(X, V), \quad(f \cdot g)_{k}=\mathrm{S}\left(\sum_{j+l=k} f_{j} \cdot g_{l}\right)
$$

The new operation is also bilinear. It is associative (commutative) if so is the initial operation.

The symmetrical composition is right distributive with respect to the multiplication, i.e., if $\varphi \in \mathcal{L}\left(X^{\prime}, X\right)$, then

$$
(f \cdot g) \stackrel{s}{\circ} \varphi=(f \stackrel{s}{\circ} \varphi) \cdot(g \stackrel{s}{\circ} \varphi) .
$$

Consider the multiplication of linear operators on a l.s. E. It can be extended to the space $\mathcal{L}_{s}\left(X, L_{1}(E)\right)$ by the formula $(1)$. The elements of the subset

$$
\mathcal{L}_{s}^{0}\left(X, L_{1}(E)\right)=\left\{\Phi=\left(\Phi_{0}, \Phi_{1}, \ldots\right) \mid \exists \Phi_{0}^{-1}\right\}
$$

form a group of invertible elements with respect to the multiplication.

If $\Phi \in \mathcal{L}_{s}^{0}\left(X, L_{1}(E)\right)$, we will denote the inverse of $\Phi$ with respect to this operation by $\Phi^{-1} \in \mathcal{L}_{s}^{0}\left(X, L_{1}(E)\right)$.

Now we define in $\mathcal{L}_{s}(X, Y)$ an operation $D_{b}: \mathcal{L}_{s}(X, Y) \rightarrow \mathcal{L}_{s}(X, Y)$ of differentiation along an element $b \in \mathcal{L}_{s}(X)$, setting

$$
\left(D_{b} a\right)_{k}=\mathrm{S}\left(\sum_{\substack{j+l=k+1 \\ j \neq 0}} \sum_{n=1}^{j} a_{j} \circ\left(\left(\bigotimes_{1}^{n-1} I_{X}\right) \otimes b_{l} \otimes\left(\bigotimes_{n+1}^{j} I_{X}\right)\right)\right) .
$$


If the spaces under consideration are topological, and $b_{0}=0$, then

$$
D_{b} a=\mathrm{S}\left(\left.\frac{d}{d \varepsilon}\right|_{\varepsilon=0} a \circ\left(i d_{X}+\varepsilon b\right)\right) .
$$

Let us denote $a \# b:=D_{b} a$. Notice that $i d_{X} \# b=b,\left(a \# i d_{X}\right)_{k}=k a_{k}$ (the Euler identity for homogeneous functions).

The operation \# is bilinear. It is not associative, but the associator

$$
a \#(b, c):=(a \# b) \# c-a \#(b \# c)
$$

is symmetric under the permutation of $b$ and $c$ :

$$
a \#(b, c)=a \#(c, b) .
$$

It is proved to be sufficient so that the following theorem should hold.

TheOREM 1. The space $\mathcal{L}_{s}(X)$ possesses a Lie algebra $\mathfrak{A}_{X}$ structure with bracket

$$
[b, c]=b \# c-c \# b .
$$

The space $\mathcal{L}_{s}(X, Y)$ is an $\mathfrak{A}_{X}$-module with action $b \mapsto D_{b}$ :

$$
\left[D_{b}, D_{c}\right] a:=\left(D_{c} D_{b}-D_{b} D_{c}\right) a=D_{[b, c]} a .
$$

Notice we can define \# by setting $a \# b=a^{\prime} \circ b$, where

$$
\begin{gathered}
': \mathcal{L}_{s}(X, Y) \rightarrow \mathcal{L}_{s}\left(X, L_{1}(X, Y)\right), \\
\left(a^{\prime}\right)_{k} h=\sum_{n=1}^{k+1} a_{k+1} \circ\left(\left(\bigotimes_{1}^{n-1} I_{X}\right) \otimes h \otimes\left(\bigotimes_{n+1}^{k+1} I_{X}\right)\right) .
\end{gathered}
$$

$\mathbf{3}^{\circ}$. We call a pair $(\mathfrak{A}, M)$, where $\mathfrak{A}$ is a Lie algebra and $M$ is $\mathfrak{A}$-module, a formal manifold. By Theorem 1 the pair $\mathfrak{M}_{X}=\left(\mathfrak{A}_{X}, \mathcal{L}_{s}(X, \mathbb{K})\right)$ forms a formal manifold. In the case under consideration, $M=\mathcal{L}_{s}(X, \mathbb{K})$ is a commutative algebra with operation of symmetric tensor multiplication - given before by formula (1). The elements of the Lie algebra $\mathfrak{A}_{X}$ act in it as differentiations.

The totality of these formal manifolds form a category $\mathfrak{M}$ with morphisms

$$
\begin{gathered}
\operatorname{Hom}\left(\mathfrak{M}_{X}, \mathfrak{M}_{Y}\right)=\mathcal{L}(X, Y), \\
\mathcal{L}(X, Y) \ni \varphi: \mathcal{L}_{s}(Y, \mathbb{K}) \rightarrow \mathcal{L}_{s}(X, \mathbb{K}), \\
f \mapsto f \stackrel{s}{\circ} \varphi .
\end{gathered}
$$

THEOREM 2. Elements $g \in G(X, Y)$ are isomorphisms of this category with action

$$
\begin{gathered}
S_{g}:\left(\mathfrak{A}_{Y}, \mathcal{L}_{s}(Y, \mathbb{K})\right) \rightarrow\left(\mathfrak{A}_{X}, \mathcal{L}_{s}(X, \mathbb{K})\right), \\
S_{g}:(A, f) \mapsto\left((g \# A) \stackrel{s}{\circ} g^{-1}=: A_{g}, f \stackrel{s}{\circ} g^{-1}=: f_{g}\right) .
\end{gathered}
$$

The following relation holds:

$$
f_{g} \# A_{g}=(f \# A) \stackrel{s}{\circ} g^{-1} .
$$

We will call the group $\mathcal{G}(X)$ the group of coordinate transformations of a formal manifold $\mathfrak{M}_{X}$.

Now we define a formal analogue of a vector bundle with $\mathfrak{M}_{X}$ as a base and a l.s. $E$ as a typical fiber. 
Theorem 3. Endow the l.s. $\mathfrak{A}_{X, E}=\mathfrak{A}_{X} \dot{+} \mathcal{L}_{s}\left(X, L_{1}(E)\right)$ with the Lie algebra structure by setting the bracket

$$
[(a, \alpha),(b, \beta)]=([a, b], \alpha \# b-\beta \# a+[\alpha, \beta]),
$$

where $[\alpha, \beta]=\alpha \cdot \beta-\beta \cdot \alpha$. Then, $\mathcal{L}_{s}\left(X, E^{*}\right)$ is an $\mathfrak{A}_{X, E}$-module with action

$$
(a, \alpha) \omega=\omega \# a+\alpha^{*} \cdot \omega .
$$

We will call the formal manifold $V(X, E)=\left(\mathfrak{A}_{X, E}, \mathcal{L}_{s}\left(X, E^{*}\right)\right)$ a total space of vector bundle with base $X$ and fiber $E$.

Every section $\xi$ determines a mapping

$$
\begin{gathered}
\tilde{\xi}: \mathcal{L}_{s}\left(X, E^{*}\right) \rightarrow \mathcal{L}_{s}(X, \mathbb{K}), \\
\tilde{\xi}: \omega \mapsto \omega \cdot \xi,
\end{gathered}
$$

so we obtain a pairing between sections of the bundle and those of the cofibration.

Coordinate transformations of the vector bundle $V(X, E)$ are defined by the pairs $(g, \Phi) \in \mathcal{G}(X) \dot{+} \mathcal{L}_{s}^{0}\left(X, L_{1}(E)\right)=: \mathcal{G}(X, E) . \mathcal{G}(X, E)$ forms a group with multiplication

$$
\left(g_{1}, \Phi_{1}\right) \cdot\left(g_{2}, \Phi_{2}\right)=\left(g_{1} \circ g_{2},\left(\Phi_{1} \stackrel{\circ}{\circ} g_{2}\right) \cdot \Phi_{2}\right) .
$$

Its identity element is the pair $\left(i d_{X}, I_{X, E}\right)$, and

$$
(g, \Phi)^{-1}=\left(g^{-1},\left(\Phi \stackrel{s}{\circ} g^{-1}\right)^{-1}\right) .
$$

The action of elements $(g, \Phi) \in \mathcal{G}(X, E)$ is defined as follows:

$$
S_{(g, \Phi)}((a, \alpha), \omega)=\left(S_{(g, \Phi)}(a, \alpha), S_{(g, \Phi)} \omega\right),
$$

where

$$
S_{(g, \Phi)}^{1}(a, \alpha)=S_{g} a
$$

and

$$
S_{(g, \Phi)}^{2}(a, \alpha)=\left[\left(\Phi \# a+\alpha^{*} \cdot \Phi\right) \cdot \Phi^{-1}\right] \stackrel{s}{\circ} g^{-1}
$$

are the components of the vector field $S_{(g, \Phi)}(A, \alpha)$, and

$$
S_{(g, \Phi)} \omega=\left(\omega \cdot \Phi^{-1}\right) \stackrel{s}{\circ} g^{-1} .
$$

THEOREM 4. $S_{(g, \Phi)}$ is a representation of $\mathcal{G}(X, E)$ concordant with $S_{g}$, i.e., the action $(g, \Phi) \mapsto S_{(g, \Phi)}$ is a homomorphism and

$$
\begin{aligned}
S_{(g, \Phi)}(f \cdot \omega) & =S_{g} f \cdot S_{(g, \Phi)} \omega, \\
S_{(g, \Phi)}(f \cdot(a, \alpha)) & =S_{g} f \cdot S_{(g, \Phi)}(a, \alpha),
\end{aligned}
$$

where $f \in \mathcal{L}_{s}(X, \mathbb{K})$

We will say that an atlas of changes of variables in a vector bundle $V(X, E)$ is introduced if a subgroup of the group $\mathcal{G}(X, E)$ is defined.

Coordinate transformations in the $\mathcal{L}_{s}(X, \mathbb{K})$-module $\mathcal{L}_{s}(X, E)$ of sections of the bundle $V(X, E)$ can be defined too, and this way we obtain a representation concordant with all the structures introduced. 
In particular, when $E=X$ we have a formal analogue of a tangent bundle on $\mathfrak{M}_{X}$, where the group of changes of variables is the subgroup of the group $\mathcal{G}(X, X)$ consisting of elements of the form $\left(g, g^{\prime}\right)$.

$4^{\circ}$. Let $\mathbf{A}(E)=\bigoplus_{r=0}^{\infty} \mathbf{A}^{r}(E)$ be the external forms algebra on a l.s. E. For $v \in E$ let $i_{v}$ be an inner product mapping:

$$
i_{v}: \mathbf{A}^{r}(E) \rightarrow \mathbf{A}^{r-1}(E), \quad i_{v}: \omega \mapsto \omega(v, \cdot, \ldots, \cdot) .
$$

Denote the 1.s. of formal $r$-forms on $V(X, E)$ by $\Omega^{r}(X, E):=\mathcal{L}_{s}\left(X, \mathbf{A}^{r}(E)\right)$. The multiplication $\wedge: \mathbf{A}^{r}(E) \times \mathbf{A}^{s}(E) \rightarrow \mathbf{A}^{r+s}(E)$ is extended to the spaces $\Omega^{r}(X, E)$ and $\Omega^{s}(X, E)$ by the formula (1). Put $\Omega(X, E):=\bigoplus_{r=0}^{\infty} \Omega^{r}(X, E)$ and extend $\wedge$ by linearity to the whole $\Omega(X, E)$. The l.s. $\Omega(X, E)$ with the multiplication $\wedge$ is called an algebra of external forms on the vector bundle $V(X, E)$.

The space of formal $r$-forms is canonically identified with the space of sets of linear mappings from $X^{\otimes k} \otimes E^{\otimes r}$ into $\mathbb{K}$ that are symmetrical in the arguments from $X$ and skew-symmetrical in the arguments from $E$ :

$$
\Omega^{r}(X, E)=\prod_{k=0}^{\infty} L_{(k, s)(r, a)}(X, E ; \mathbb{K}) .
$$

We will separate the symmetrical and skew-symmetrical arguments with a vertical line: if $\omega \in \Omega^{r}(X, E)$, then

$$
\omega_{k}\left(x_{1}, \ldots, x_{k}\right)\left(h_{1}, \ldots, h_{r}\right)=\omega\left(x_{1}, \ldots, x_{k} \mid h_{1}, \ldots, h_{r}\right) .
$$

Thus, if $\omega \in \Omega^{r}(X, E), \theta \in \Omega^{r}(X, E)$, then

$$
\begin{aligned}
&(\omega \wedge \theta)_{k}\left(x_{1}, \ldots, x_{k} \mid h_{1}, \ldots, h_{r+s}\right)= \\
&=\sum_{j+l=k}\left(\omega_{j} \otimes \theta_{l}\right) \circ(\overbrace{x_{1}, \ldots, x_{j}} \mid \underbrace{h_{1}, \ldots, h_{r}}) \otimes(\overbrace{x_{j+1}, \ldots, x_{k}} \mid \underbrace{h_{r+1}, \ldots, h_{r+s}}),
\end{aligned}
$$

where the top braces denote symmetrization in the corresponding elements and the bottom braces alternation.

The inner product is extended to the sections of the bundle and external forms by the formula (1):

$$
\left(i_{\xi} \omega\right)_{k}=\mathrm{S}\left(\sum_{j+l=k} \omega_{j} \circ\left(\left(\bigotimes_{1}^{j} I_{X}\right) \mid \xi_{l} \otimes\left(\bigotimes_{2}^{r} I_{E}\right)\right)\right)
$$

where $\xi \in \mathcal{L}_{s}(X, E), \omega \in \Omega^{r}(X, E)$, with

$$
i_{\xi}(\omega \wedge \theta)=\left(i_{\xi} \omega\right) \wedge \theta+(-1)^{r} \omega \wedge\left(i_{\xi} \theta\right),
$$

where $r$ is the power of the form $\omega$.

Denote the change of variables in $\Omega(X, E)$ by the same symbol $S_{(g, \Phi)}$ :

$$
S_{(g, \Phi)} \omega=\left(\omega \cdot\left(\Phi^{-1}\right)^{\otimes r}\right) \stackrel{s}{\circ} g^{-1},
$$


where $r$ is the power of the form $\omega$. The change of variables is proved to be concordant with all the operations.

Let us introduce the de Rham complex. Consider the algebra of external forms on the manifold $\mathfrak{M}_{X}, \Omega(X):=\Omega(X, X)$. Define a mapping $d: \Omega^{r}(X) \rightarrow \Omega^{r+1}(X)$ by

$$
(d \omega)_{k}\left(x_{1}, \ldots, x_{k} \mid h_{1}, \ldots, h_{r+1}\right)=(k+1) \omega_{k+1}(x_{1}, \ldots, x_{k}, \underbrace{h_{1} \mid h_{2}, \ldots, h_{r+1}}) .
$$

Extend $d$ to the whole $\Omega(X)$ by linearity. It satisfies to all the usual properties of external differential, and, in particular,

$$
\begin{aligned}
(d \omega)\left(A_{1}, \ldots, A_{r+1}\right)= & \sum_{i=1}^{r+1}(-1)^{i-1} A_{i} \omega\left(A_{1}, \ldots, \hat{A}_{i}, \ldots, A_{r+1}\right)+ \\
& \sum_{i<j}(-1)^{i+1} \omega\left(\left[A_{i}, A_{j}\right], A_{1}, \ldots, \hat{A}_{i}, \ldots, \hat{A}_{j}, \ldots, A_{r+1}\right),
\end{aligned}
$$

where $\omega \in \Omega^{r}(X), A_{j} \in \mathfrak{A}_{X}, \quad j=1, \ldots, r+1$, and the hat denotes the absence of a symbol.

The exact and closed forms are defined in the usual way. In particular, the exact 1forms are distinguished among all the 1-forms by the property that they are symmetrical in all the arguments.

Define a linear mapping $\delta: \Omega^{r+1}(X) \rightarrow \Omega^{r}(X)$ by

$$
(\delta \omega)_{k}\left(x_{1}, \ldots, x_{k} \mid h_{1}, \ldots, h_{r}\right)=\frac{1}{k} \omega_{k-1}(\overbrace{x_{1}, \ldots, x_{k-1} \mid x_{k}}, h_{1}, \ldots, h_{r}),
$$

and extend it to $\Omega X$ by linearity. The properties of $d$ hold for $\delta$. The $\{\delta, d\}=\delta d+d \delta$ is differentiation of power 0 of the algebra $\Omega(X)$ (see $[7, \mathrm{Ch}$. IV]).

Proposition 3. If $\omega \in \Omega^{r}(X)$, then

$$
(\{\delta, d\} \omega)_{k}=(r+k) \omega_{k} .
$$

A Lie differentiation of the external form $\omega$ along the vector field $A$ can be defined by the formula $L_{A} \omega=\left\{i_{A}, d\right\} \omega$.

$5^{\circ}$. We consider in this item linear topological spaces.

Consider a 1-parameter family $a(t) \in \mathcal{L}(X)$ of vector fields. If there exists a 2parameter family $u(t, \tau) \in \mathcal{L}_{s}(X)$ such that

$$
\frac{d u(t, \tau)}{d t}=a(t) \stackrel{s}{\circ} u(t, \tau), \quad u(\tau, \tau)=i d,
$$

than $a(t)$ is called to be integrable. In this case $u(t, \tau)$ is evolutionary family, i.e.,

$$
u(t, \tau)=u(t, s) \stackrel{s}{\circ} u(s, \tau) .
$$

$u(t, \tau)$ is called the evolutionary family of shifts along the trajectories of $a(t), a(t)$ is called the generator of the family $u(t, \tau)$. The generator can be found by the usual formula

$$
a(t)=\left.\frac{d}{d s} u(s, t)\right|_{s=t} .
$$

It is proved that $a(t)$ is integrable if and only if its linear part $a_{1}(t)$ is integrable, with $u_{2}(t, \tau), \ldots$ calculated by the explicit formulae (see $\left.[3]\right)$. 
Consider the evolutionary family generated by a constant formal vector field $a$. In this case equation (3) is invariant with respect to the shifts $t \mapsto t-s$. It means $u(t, \tau)=u(t-\tau)$ depends only on the difference of the arguments. Further, the family $u(t) \quad(t \geq 0)$ forms a semigroup because (3) has the form

$$
u\left(t_{1}+t_{2}\right)=u\left(t_{1}\right) \circ u\left(t_{2}\right) \quad\left(t_{1}, t_{2} \geq 0\right), \quad u(0)=i d_{X} .
$$

We call the family $u(t)$ a composite exponential and denote it by

$$
\stackrel{\circ}{\exp } a t=u(t)
$$

Proposition 4. The composite exponential satisfies the following relation (see [1]):

$$
f \stackrel{s}{\circ} \underset{\exp }{\exp } a t=e^{t D_{a}} f
$$

where $f \in \mathcal{L}_{s}(X, Y)$ is an object on $X$, and $e^{t D_{a}}$ is the semigroup of linear operators on $\mathcal{L}_{s}(X, Y)$ with the generator $D_{a}$

So, it is sufficient to calculate the linear exponential $e^{t D_{a}}$ for calculation the composite one (see [3]).

$6^{\circ}$. Define a connection on the v.b. $V(X, E)$ with the help of the formal mapping $\Gamma \in \mathcal{L}_{s}\left(X, L_{1}\left(X, L_{1}(E)\right)\right)$ called a coefficient of connection or the Christoffel symbol.

A coefficient of connection defines a covariant derivative of the section $\xi$ of the bundle $V(X, E)$ along the vector field $\eta$ :

$$
\begin{gathered}
\nabla^{\Gamma}: \mathfrak{A}_{X} \dot{+} \mathcal{L}_{s}(X, E) \rightarrow \mathcal{L}_{s}(X, E), \\
\nabla_{\eta}^{\Gamma} \xi=\xi \# \eta+(\Gamma \cdot \eta) \cdot \xi .
\end{gathered}
$$

The transformation

$$
S_{(g, \Phi)}: \Gamma \mapsto \Gamma_{(g, \Phi)}=\left[\left(\left(\Phi \cdot \Gamma-\Phi^{\prime}\right) \cdot\left(g^{-1}\right)^{\prime}\right) \cdot \Phi^{-1}\right] \stackrel{s}{\circ} g^{-1}
$$

of the connection coefficient corresponds to the coordinate transformation $(g, \Phi)$ of the bundle.

Proposition 5. The following relations hold

$$
\begin{gathered}
\nabla_{f \cdot \eta}^{\Gamma} \xi=f \cdot \nabla_{\eta}^{\Gamma} \xi, \\
\nabla_{\eta}^{\Gamma} f \cdot \xi=f \# \eta+f \cdot \nabla_{\eta}^{\Gamma} \xi, \\
S_{(g, \Phi)}^{*} \nabla_{\eta}^{\Gamma} \xi=\nabla_{\eta_{g}}^{\Gamma_{(g, \Phi)}} \xi_{(g, \Phi)}, \\
-\Gamma_{(g, \Phi)} \cdot \eta_{g}=S_{(g, \Phi)}^{2}(\eta,-\Gamma \cdot \eta),
\end{gathered}
$$

where $S_{(g, \Phi)}^{2}$ acts by formula (2).

We say a section $\xi$ of the v.b. $V(X, E)$ is parallel along the trajectories of a vector field $\eta$ if the following relation holds:

$$
\nabla_{\eta}^{\Gamma} \xi=0
$$

We further consider linear topological spaces. 
Suppose that vector field $\eta$ without zero term generates an evolutionary family $u(t, \tau)$ $\in \mathcal{L}_{s}(X)$ of shifts along the trajectories of $\eta$ :

$$
\frac{d u(t, \tau)}{d t}=\eta \stackrel{s}{\circ} u(t, \tau), \quad u(\tau, \tau)=i d .
$$

Proposition 6. The linear differential equation

$$
\frac{d U(t, \tau)}{d t}=-[(\Gamma \cdot \eta) \stackrel{s}{\circ} u(t, \tau)] \cdot U(t, \tau), \quad U(\tau, \tau)=I_{X, E}
$$

together with (4) defines the evolutionary pair $(u, U) \in \mathcal{L}_{s}(X) \times \mathcal{L}_{s}\left(X, L_{1}(E)\right)$ of parallel translation along the trajectories of the $\eta$ possessing the property

$$
(U(t, s) \stackrel{s}{\circ} u(s, \tau)) \cdot U(s, \tau)=U(t, \tau), \quad t \geq s \geq \tau .
$$

The evolutionary pair is invariant with respect to the changes of variables, i.e., the pair $\left(u_{g}, U_{(g, \Phi)}\right)$ specifies the parallel translation along $\eta_{g}$ in the connection $\Gamma_{(g, \Phi)}$.

THEOREM 5. The section $\xi \in \mathcal{L}_{s}(X, E)$ possessing the property

$$
\xi \stackrel{s}{\circ} u(t, \tau)=U(t, \tau) \cdot \xi
$$

is parallel along the $\eta$.

\section{References}

[1] M. N. Araslanov, Yu. L. Daletskiı̌, Composition Logarithm in the Class of Formal Operator Power Series, Funct. Anal. Appl. 26 (1992), 57-60.

[2] A. M. Baranovitch, Yu. L. Daletskiǐ, Differential-Geometric Relations for Formal Operator Power Series Class, Preprint.

[3] Yu. L. Daletskiǐ, Algebra of Compositions and Non-Linear Equations, appear.

[4] Yu. L. Daletskǐ̌, S. V. Fomin, Measures and Differential Equations in Infinite-Dimensional Space, Kluwer Acad. Publ., Dordrecht/Boston/London, 1991.

[5] I. M. Gel'fand, Yu. L. Daletskiř, B. Tsygan, On a Variant of Non-Commutative Differential Geometry, Doklady Academii Nauk USSR 308 (1989), 422-425.

[6] M. Gerstenhaber, The Cohomology Structure of an Associative Ring, Ann. Math 78 (1963), 59-103.

[7] C. Godbillon, Géométrie Différentielle et Mécanique Analytique, Hermann, Paris, 1969. 